\title{
Fabrication of PEDOT coated PVA-GO nanofiber for supercapacitor
}

\begin{abstract}
Conducting nanofibers comprised of poly(vinyl alcohol) (PVA)-graphene oxide (GO) nanofiber coated with poly(3,4-ethylenedioxythiophene) (PEDOT) for supercapacitor application was prepared through integrated techniques i.e. electrospinning and electrodeposition. The formation of smooth cross-linking nanofibers without beads proved that GO has uniformly distributed into PVA with an average diameter of $117 \pm 32 \mathrm{~nm}$. Field emission scanning electron microscopy (FESEM) images revealed that cauliflower-like structure of PEDOT grew well on the surface of PVA-GO nanofibers with high porosity. Fourier transform infrared spectroscopy (FTIR) and Raman spectroscopy proved the existence of PVA, GO, and PEDOT. PVA-GO/PEDOT nanocomposite showed the highest specific capacitance $(224.27 \mathrm{~F} / \mathrm{g})$ compared to PEDOT $(167.92 \mathrm{~F} / \mathrm{g})$ and PVA/PEDOT (182.73 F/g). PVA-GO/PEDOT nanocomposite exhibited $1.8 \mathrm{~V}$ wide operating potential windows which significantly can enhance its capacitive behaviour. PVA-GO/PEDOT nanocomposite has also demonstrated superior performance with the energy density and power density of $9.58 \mathrm{Wh} / \mathrm{kg}$ and $304.37 \mathrm{~W} / \mathrm{kg}$, respectively at $1.0 \mathrm{~A} / \mathrm{g}$ current density. PVAGO/PEDOT nanocomposite revealed the smallest resistance of charge transfer (Rct) and equivalent series resistance (ESR) indicating excellent charge propagation behaviour at the interfacial region. The composite exhibits a good capacity retention of $82.41 \%$ after $2000 \mathrm{CV}$ cycles and further drops $11.27 \%$ after 5000 cycles caused by the swelling and shrinkage of the electrode material during the charging and discharging processes.
\end{abstract}

Keyword: PVA nanofibers; Graphene; Poly(3,4-ethylenedioxythiophene); Electrospinning; Supercapacitor 